\title{
Clinical trial participation poses ethical, practical issues
}

I $t$ could be called one of the dark and enduring secrets of modern practice.

Several physicians say, on condition of absolute anonymity, that they earn tens of thousands of dollars annually by recruiting patients

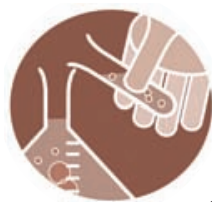
for clinical trials. One even admits that he's earned as much as $\$ 2000$ from a pharmaceutical firm for each patient that he persuaded to participate in a clinical trial of a cancer drug.

Is that peanuts? Are others earning even more?

Who knows?

Ask a physician for an on-the-record comment about any manner of patient recruitment fees and the invariable response is that physician participation in clinical trials is a necessary to advance knowledge and improve patient care. That, or to cite "confidentiality" as an excuse for silence.

Yet many physicians are increasingly concerned about the pressures to commercialize research, which raises potential conflicts of interest, and for some, ethical quandaries about receiving monies to boost patient enrolments in clinical trials. Still others raise concerns about patient safety and welfare.

When pharmaceutical firms, which fund the majority of clinical trials in Canada, chase physicians to recruit patients, the doctors often have questions about what they are being asked to do, says Dr. Jeff Blackmer, executive director of the Canadian Medical Association's office of ethics. Those queries typically come from doctors in community (nonacademic) settings: Is participation ethical? Is the sponsor offering too much money? Should the physician agree to sign a nondisclosure form?

The array of fees is often dazzling, from flat fees for simply recruiting a patient to finders' fees and completion fees (Box 1).

The fees are viewed as a necessary condition for physician involvement which, in turn, is necessary for patient recruitment. The driver, of course, is profits from pharmaceutical sales.

The upshot, though, is that physi- cians who engage in clinical research must juggle an array of issues - patients' expectations, trial transparency, disclosure of results, scientific validity, trial merits, patient risks, and, of course, financial opportunities contained therein.

The growing raft of ethical concerns surrounding physician involvement in trials has prompted some organizations to weigh in on the debate.

Canada's 3 granting councils, for example, recently released a new draft of their ethical guidelines for research involving humans (CMAJ 2009;180 [4]:379-80 and www.pre.ethics.gc.ca /english/pdf/newsandevents/TCPS_Dec _4_en.pdf) that includes a new section on financial conflicts of interest involving physicians. It instructs research ethics boards within universities to scrutinize clinical trial budgets for "unreasonable payments and undue inducements," such as finders' fees for referring patients.

It's a "very positive development," says Lorraine Ferris, professor of public health at the University of Toronto in Toronto, Ontario.

Because there is a divergence between the physician's private interests and his or her obligations to the patient, the University of Toronto's Faculty of Medicine began 6 years ago to prohibit the offer or acceptance of finders' and completion fees. Meanwhile, the College of Physicians and Surgeons of Ontario in 2006 developed a policy concerning conflicts of interest and patient recruitment for clinical trials, under which noncompliance with its provisions may constitute professional misconduct (cpso.on.ca /policies/policies/default.aspx?ID=1536).

A commentary that Ferris coauthored with Dr. C. David Naylor (CMAJ 2004; 171[8]:883-6) calls for a standardized template for clinical trial budgets, and for transparency about those budgets, to facilitate disclosure and promote equity in physicians' clinical trial remuneration. These ideas, however, have not yet gained traction in the wider research community.

As a consequence, oversight is at best sporadic. As Muhammad Mamdani, director of the applied health research centre at the Li Ka Shing Knowledge Institute of St. Michael's Hospital in Toronto, notes, issues around physician remuneration and involvement in clinical trials in Canada are mostly governed by guidelines "and a lot of guidelines are not very proscriptive. There is too much room to play."

Various groups have proposed solutions to promote a more unified approach, but no one body has emerged with enough authority and funding to compel the creation of such a creature.

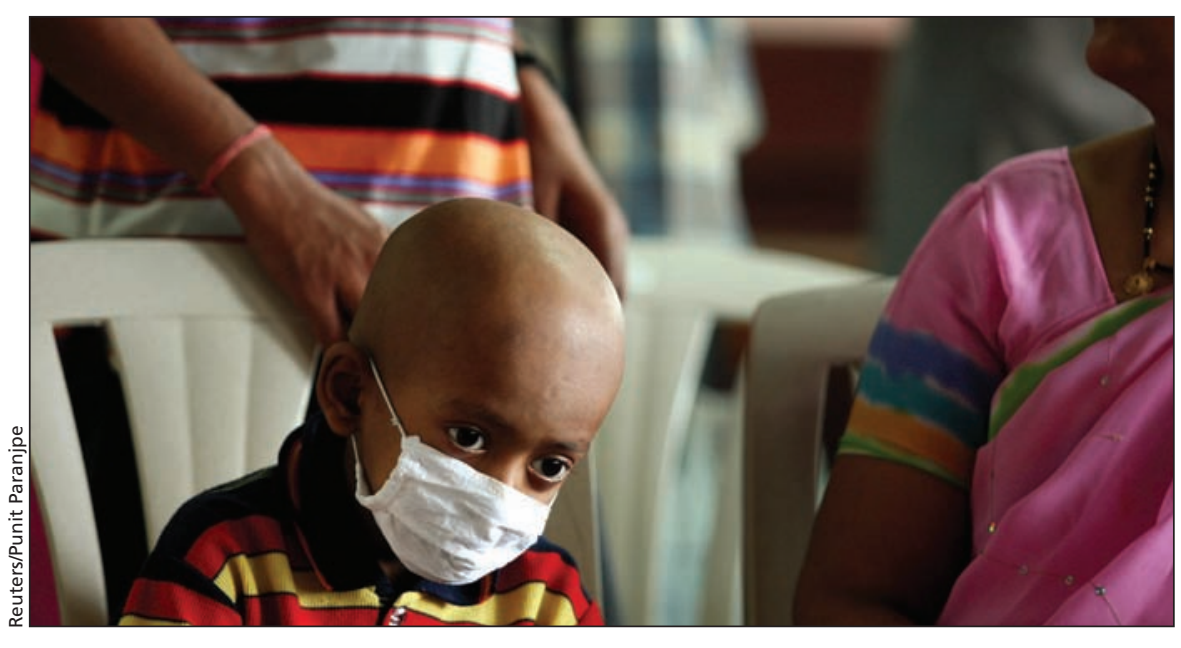

The growing demand for clinical trial participants has led pharmaceutical companies to look overseas, and controversy has followed. It has been estimated that in India, where some people sign consent forms they can't read or are enrolled in trials without their knowledge, there will be about 2 million clinical trial patients by 2010 . 


\section{Shift to community}

The past decade has seen most of the clinical trial activity in Canada shift away from academic hospitals and institutions into the community (CMAJ 2004;170[1]:33), where ethics reviews are primarily conducted by independent research ethics boards, which are not obliged to follow the Tri-Council policy. It applies only to institutions that receive funding from the Canadian Institutes for Health Research, Social Sciences and Humanities Research of Canada and Natural Sciences and Engineering Research Council of Canada.

The result is that ethics boards vary in their evaluation of trials - so much so that it is not unheard of for the managers and sponsors of clinical trials to go shopping for a more positive evaluation, says Blackmer, whose office recently developed detailed research guidelines for physicians (policybase.cma.ca /dbtw-wpd/Policypdf/PD08-01.pdf).

In academic settings, doctors with queries already have access to other resources, such as local research ethics board or vice-presidents of research, Blackmer adds.

For example, the ethics board at St. Michael's Hospital "works very hard to be available" and has set up a consultation system to direct physicians' questions about science, ethics and payment to the appropriate experts, says Dr. Brenda McDowell, vice-chair of the board. About $60 \%$ of the clinical trials that the 15-18-member board scrutinizes annually are sponsored by industry, she says.

Some specialists have banded together to share clinical trial expertise. The 15-year-old Canadian Stroke Consortium has 170 members and 50 clinical sites and hence provides "one-stop shopping" for pharmaceutical companies, says business manager Donna $\mathrm{Hu}$ ber. A company pays the consortium to review its trial protocol, and the doctors' group decides whether or not to endorse the trial and/or to seek changes. Huber then negotiates a per-patient budget — standard across all the sites.

Cooperative groups led by academics are another feature of the clinical trial landscape. "We provide a platform for a coordinated approach," says Dr. Ralph Meyers, who heads the clini- cal trials group at the National Cancer Institute of Canada. For example, the Institute can coordinate multiple trials for different populations suffering from the same cancer. When Meyers' group conducts trials in collaboration with industry, it manages the data and sponsors the trial at a regulatory level, he says. "As you can imagine with new anticancer drugs, you have to collaborate with industry, because it's the only way of getting new agents."

\section{Attitudes and concerns differ}

Physicians' concerns about the ethics involved in research trials often depend upon the type of trial being proposed and the research setting.

The source of the industry funding is also a factor, says Mamdani, the St. Michael's director of applied health research. If the money is coming from a company's medical affairs department, "there is a bit more of a process around scientific rigour," he says. "If the funding is from marketing, no one really knows what the rules are."

Mamdani, a pharmacist who spent a year as director of outcomes research at Pfizer Global Pharmaceuticals, says there are essentially 3 types of industryfunded clinical trials:

- investigator-initiated trials, where the investigator has a protocol and is given funding to conduct the trial without company involvement;

- trials where the company writes the protocol and designs the trial; and

- trials where a company takes a keen interest in an investigator's idea and adopts, expands and shapes it.

Investigator-initiated trials are probably a diminishing proportion of the total since "I think industry wants more control," says Mamdani. Moreover, industry sometimes funds these trials without caring about their outcomes for the purpose of "relationship building" with a key investigator, he adds.

The attitude and interests of doctors based in the community and those in academic settings are different, says Martin Letendre, director of ethics and legal affairs for ethica Clinical Research Inc., a Montréal, Quebec-based contract research organization. Academic physicians may have their own research agenda and want to publish.

\section{Box 1: Definitions}

Fees prohibited by the University of Toronto Faculty of Medicine:

Finders' fees: Money or other reward given by the sponsor (or by a physician) to a physician (or a group of physicians) in payment for identifying or recruiting a patient into a study or trial. Finders' fees include bonus or milestone payments for successfully enrolling a particular number of patients or for successfully meeting a deadline in recruiting patients.

Completion fees: Money or other reward given by the sponsor (or by a physician) to a physician (or a group of physicians) in payment for each patient's successful completion of the study or trial protocol. Completion fees include bonus or milestone payments for a particular number of patients successfully completing the study or trial or for successfully completing it within a specific time frame.

Source: University of Toronto.

They are more likely to be seasoned investigators who are less interested in conducting a trial using a protocol a company has written for them. Meanwhile, those who work on trials with a sponsor-written protocol can be seen as essentially "implementers."

\section{Research or marketing?}

Letendre asserts that when his company manages a clinical trial, it is interested in using "seasoned investigators with good track records" who treat patients fairly, meet recruitment targets and provide quality data.

In contrast, he says, when drug companies sponsor trials and recruit investigators, they often seek high prescribers and community leaders with whom they are familiar from medical meetings, or those who they have received information about from their marketing departments. This approach is particularly true of phase 4 trials of drugs that Health Canada has already approved, he adds. (CMA policy suggests that physicians avoid becoming involved in trials that are clearly intended for marketing.)

In general, research is increasingly "integrated within a complex marketing 
scheme" and companies compete to create links with physicians who can recruit patients, conduct studies, lecture about new products and "provide overall credibility" to industry-sponsored research, University of Toronto law professor Trudo Lemmens states in a draft document submitted to the bioethics education program of the Royal College of Physicians and Surgeons of Canada.

\section{Costs and payment}

In academic settings, about $30 \%$ of a clinical trial budget goes to pay overhead costs, says Dr. Penny MoodyCorbett of the Faculty of Medicine at Memorial University in St. John's, Newfoundland and Labrador. She adds that the common perception that trials are a money-maker for institutions is errant. At Memorial, "they have not been a lucrative activity ... that is our experience."

Whether physicians are remunerated for time spent on clinical trials appears conditional on institution and discipline. Meyers says no extra payment goes to physicians who are on salary and working in the field of cancer. Rather, the monies are used to pay for clinical research assistants and staff, and to pay hospitals for extra tests and procedures the trial requires.

CMA guidelines say that physician compensation for trials work should be over and above their usual care for patients. Blackmer says it should also be commensurate with expenses incurred and that research subjects must be told if their doctor is receiving a fee for their participation in a trial, and "by whom the fee will be paid."

Regardless of their motive for getting involved in clinical trials, doctors should consider the time commitment involved.

It is useful to have family doctors recruit patients in order to get a general sample of the population, McDowell says. But she'd look "long and hard" at whether she could meet the requirement for trial involvement if she had a busy clinical practice.

In academic centres, doctors express frustration about issues such as the speed of research ethics board approval, resource management and contract arrangements, says Moody-Corbett.
Since it is "not in our interests to discourage researchers," her university has taken steps to streamline processes.

At the end of the day, most doctors really want to do the right thing for their patients, says Mamdani. "I think the money factor is important, but ... physicians have to feel this is an important issue they are dealing with clinically and buy into the trial. Otherwise, they are just doing it for money ... and I bet in those cases, recruitment rates aren't going to be that high." - Ann Silversides, CMAJ

DOI:10.1503/cmaj.090131
This article is part of a series on clinical trials that the CMAJ News section will run throughout 2009. Previous articles included an overview of the landscape of trials in Canada (CMAJ 2009;180[1]:20-22); a short history of trials (CMAJ 2009;180[1]:234); an article on the rising costs of trials (CMAJ 2009;180[3]:277-78); an article on drug development costs (CMAJ 2009;180[3]:279-80) and an article on recruiting patients (CMAJ 2009;180[4]: 375-8). Future articles will explore registration, ethical oversight, patient safety, reporting and the push for reforms.

\section{Academy pitches new approach for evaluating research investments}

W ith accountability having become the latest bureaucratic and political buzzword in Ottawa, research agencies are under increasing pressure to develop measures to ensure that tax dollars are spent wisely, while simultaneously demonstrating the value of a tax dollar invested in research, or in one specific discipline as opposed to another.

Are there more dividends in investing in home care research or directly in home care?

Is a tax dollar invested in health research more likely to yield economic and social dividends than a dollar invested in astronomy? Or even prove more costly to government coffers because research is a "cost-driver" of the health care system?

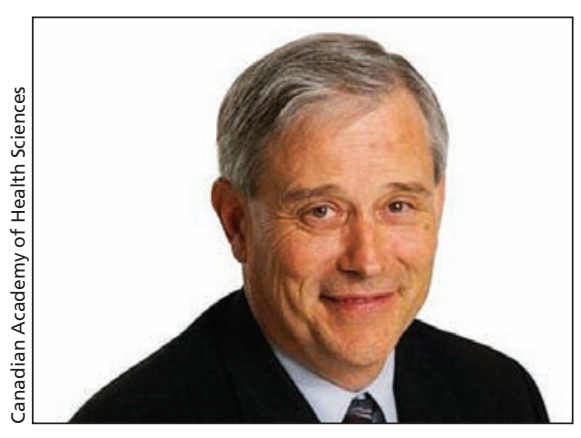

Canada could become an international leader in measuring the value of research investments, says Dr. Cyril Frank.
How should a department or agency determine priority areas of research investment?

The answers are as complex as the questions, the Canadian Academy of Health Sciences said while releasing the findings of its first major study since being founded in 2004 as a nonprofit organization providing independent advice to governments and interested parties. The $\$ 500000$ study was sponsored by 23 organizations.

The study's final report, Making an Impact: A Preferred Framework and Indicators to Measure Returns on Investment in Health Research, urges that governments, industry and national organizations adopt a new framework for evaluating and prioritizing research investments (www.cahs-acss.ca/e/pdfs /ROI_FullReport.pdf).

The framework uses a "systems approach" to measure return on investment and is based on a "payback model," developed by Martin Buxton and the Health Economics Research Group at Brunel University in the United Kingdom, under which research investments or programs are evaluated in 5 categories: "knowledge, benefits to future research, political and administrative benefits, health sector benefits, and broader economic benefits."

The framework was crafted by an international panel chaired by Dr. Cyril 